\title{
Genotype-phenotype correlation in 75 patients with small supernumerary marker chromosomes
}

Tingting Li ${ }^{1}$, Haiquan Sang ${ }^{2}$, Guoming Chu', Yuanyuan Zhang ${ }^{1}$, Manlong Qi', Xiaoliang Liu', Wanting Cui and Yanyan Zhao ${ }^{1 *}$

\begin{abstract}
Background: Small supernumerary marker chromosomes (sSMCs) are rare structural abnormalities in the population; however, they are frequently found in children or fetuses with hypoevolutism and infertile adults. SSMCs are usually observed first by karyotyping, and further analysis of their molecular origin is important in clinical practice. Nextgeneration sequencing (NGS) combined with Sanger sequencing helps to identify the chromosomal origins of sSMCs and correlate certain SSMCs with a specific clinical picture.

Results: Karyotyping identified 75 sSMCs in 74,266 samples (0.1\% incidence). The chromosomal origins of 27 of these sSMCs were detected by sequencing-related techniques (NGS, MLPA and STR). Eight of these sSMCs are being reported for the first time. SSMCs mainly derived from chromosomal $X, Y, 15$, and 18, and some SSMC chromosomal origins could be correlated with clinical phenotypes. However, the chromosomal origins of the remaining 48 sSMC cases are unknown. Thus, we will develop a set of economical and efficient methods for clinical SSMC diagnosis.

Conclusions: This study details the comprehensive characterization of 27 sSMCs. Eight of these SSMCs are being reported here for the first time, providing additional information to SSMC research. Identifying SSMCs may reveal genotype-phenotype correlations and integrate genomic data into clinical care.
\end{abstract}

Keywords: Small supernumerary marker chromosomes, Next-generation sequencing, Prenatal diagnosis, Genetic counseling

\section{Background}

Small supernumerary marker chromosomes (sSMCs) are structural abnormalities whose origins cannot be characterized by conventional cytogenetics alone but require molecular approaches. It is known that $70 \%$ of sSMCs are de novo, $20 \%$ are inherited from the mother, and $10 \%$ come from the father [1]. sSMCs are often derived from maternal meiosis I/II errors, trisomic/monosomic rescue, or fertilization errors $[2$,

\footnotetext{
* Correspondence: yyzhao@sj-hospital.org

${ }^{1}$ Department of Clinical Genetics, Shengjing Hospital of China Medical University, 36 Sanhao Street, Heping District, Shenyang 110003, China

Full list of author information is available at the end of the article
}

3]. sSMCs are equal to or smaller than chromosome 20 in size and often have abnormal morphology (e.g., inverted duplication, minute, or ring). Many of them are derived from the short arms or pericentromeric regions of chromosomes. Nearly 70\% of sSMC carriers are clinically normal; however, $30 \%$ are abnormal. Patients carrying sSMCs have developmental delays, intellectual disabilities, mixed gonadal dysgenesis (MGS), or infertility, depending on the origin of the sSMC. The treatment of these patients was based on different symptoms until the molecular characterization of sSMCs was developed.

(c) The Author(s). 2020 Open Access This article is licensed under a Creative Commons Attribution 4.0 International License, which permits use, sharing, adaptation, distribution and reproduction in any medium or format, as long as you give appropriate credit to the original author(s) and the source, provide a link to the Creative Commons licence, and indicate if changes were made. The images or other third party material in this article are included in the article's Creative Commons licence, unless indicated otherwise in a credit line to the material. If material is not included in the article's Creative Commons licence and your intended use is not permitted by statutory regulation or exceeds the permitted use, you will need to obtain permission directly from the copyright holder. To view a copy of this licence, visit http://creativecommons.org/licenses/by/4.0/ The Creative Commons Public Domain Dedication waiver (http://creativecommons.org/publicdomain/zero/1.0/) applies to the data made available in this article, unless otherwise stated in a credit line to the data. 
In this study, we identified $75 \mathrm{sSMC}$ cases in 74,266 patients seen in our department from 2015 to 2018 by karyotyping. Fifty-seven of the cases were subjected to molecular analysis, and the remaining 18 were not characterized further. Next-generation sequencing (NGS) is a fast high-output sequencing technique used to determine copy number variations [4]. We combined NGS, multiplex ligation-dependent probe amplification (MLPA), and short tandem repeat (STR) analysis to identify the origins of the sSMCs in our study. The molecular components of 27 of the sSMCs were identified. Thirty of the sSMCs subjected to molecular analysis did not have any pathogenic information in original chromosomal.

sSMCs were first detected by conventional cytogenetic banding analysis, which is weak for identifying their molecular component. This study aimed to identify the origins of sSMCs diagnosed in our department over the last 4 years. This application may help recognize syndromes from which sSMC patients suffer, establish suitable and specific therapy, or even predict syndromes that will develop in the future. Such an application will be of great value in clinical genetic diagnosis and genetic counseling.

\section{Results}

\section{Distribution of cases}

A total of 74,266 samples were analyzed for genetic diagnosis from the infertility, pediatrics, and obstetrics departments of Shengjing hospital (Fig. 1). In particular, we studied 75 sSMC carriers $(0.1 \%$ in total), including
23 adults with infertility or habitual abortion (23/75, $30.67 \%), 20$ children with severe developed delay, MGS or gynandromorphism $(20 / 75,26.67 \%), 23$ fetuses with intrauterine growth retardation or abnormal ultrasonic structures $(23 / 75,30.67 \%)$, and nine unsyndromatic sSMC cases $(9 / 75,12 \%)$. We performed NGS, MLPA, and STR on 57 sSMCs and identified the chromosomal origins for 27 of these cases (Table 1). The chromosomal origins of the remaining 48 cases are still unknown (Table 2). These data suggested that most SSMC cases have clinical syndromes, which might be correlated with their clinical phenotypes.

\section{sSMCs from chromosome $Y$}

Twelve sSMCs were derived from chromosome Y. Patients 61166 and W02938 were sexually abnormal boys, showing similar characteristics to Turner syndrome with androgynous. Results showed the sSMCs were derived from a minute $Y$ chromosome with SRY (Fig. 2A, B). Patient 69433 grew up as a girl. The MLPA analysis indicated that the SSMC was derived from $\min (\mathrm{Y})$ (Fig. 2C). Patients 61680, 62091, 77297, 80794, 98139 and W01824 were adult men with azoospermia and infertility. STR analysis showed that their sSMCs came from $\min (\mathrm{Y})$ (Fig. 2D-I, Table 3). Samples 150677, 162047, and 171276 were from amniotic fluid. The STR analysis results demonstrated that the sSMCs were from $\min (\mathrm{Y})$ (Fig. 2J-L).
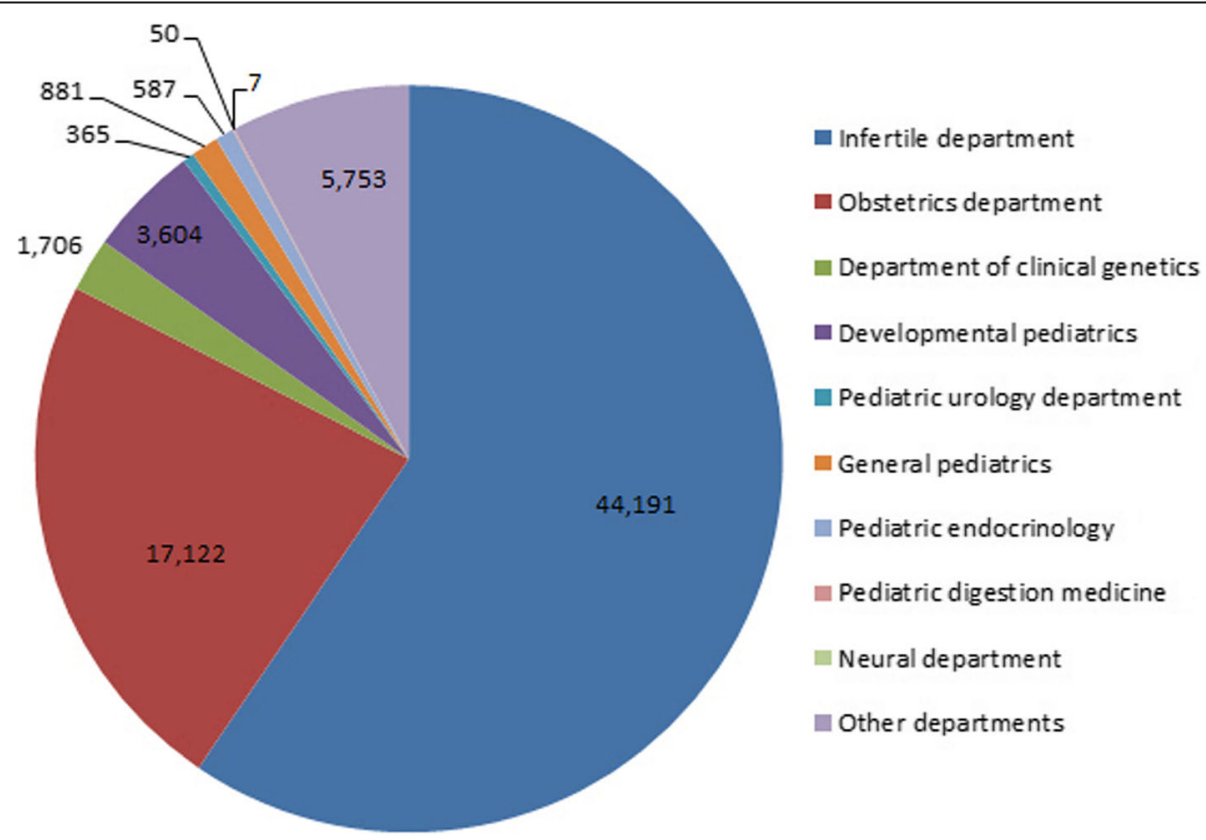

Fig. 1 Departmental distribution of patients in the last 4 years. The patients in this study came mainly from the infertility (44,191), obstetrics $(17,122)$, and developmental pediatric $(3,604)$ departments of Shengjing hospital 
Table 1 The information of 27 identified SSMC patients

\begin{tabular}{|c|c|c|c|c|c|c|c|c|}
\hline $\begin{array}{l}\text { Patient } \\
\text { NO. }\end{array}$ & $\begin{array}{l}\text { Gender/ } \\
\text { age at } \\
\text { diagnosis }\end{array}$ & $\begin{array}{l}\text { Studied } \\
\text { material }\end{array}$ & Cytogenetics & Final result of the sSMC & Test methods and results & Clinical symptoms & $\begin{array}{l}\text { Age of } \\
\text { gravida/ } \\
\text { karyotypes } \\
\text { of parents }\end{array}$ & $\begin{array}{l}\text { De } \\
\text { novo/ } \\
\text { inherited }\end{array}$ \\
\hline $61166^{\mathrm{a}}$ & $\begin{array}{l}\text { male/14 } \\
\mathrm{m}\end{array}$ & PBL & $\begin{array}{l}45, X[2] / 46, X \\
+\operatorname{mar}[15]\end{array}$ & $\begin{array}{l}\text { del }(Y)(\text { pter } \rightarrow \text { q11.222:: } \\
\text { q11.223 } \rightarrow \text { qter }), \text { first } \\
\text { report }\end{array}$ & $\begin{array}{l}\text { NGS:del(Y)(p11.2)×0.5 (2.7 Mb), } \\
\text { del(Y)(q11.222 } \rightarrow q 11.223) \times 0(2.2 \\
\text { Mb). AZF b, - d and -c regions: } \\
\text { deleted. STR:AMEL (Xp22.2: } \\
\text { Yp11.2): 2:1. SRY (Yp11.31): } \\
\text { positive. }\end{array}$ & $\begin{array}{l}\text { Hypospadias, right cryptorchidism, } \\
\text { term birth (BW } 2.15 \mathrm{~kg} \text { ). He } \\
\text { performed the corrective surgery } \\
\text { before karyotyping report. }\end{array}$ & n.a. & n.a. \\
\hline W02938 & $\begin{array}{l}\text { male/13 } \\
\mathrm{m}\end{array}$ & PBL & $\begin{array}{l}45, X[26] / 46 \\
X,+\operatorname{mar}[19]\end{array}$ & $\min (Y)$ with SRY & $\begin{array}{l}\text { STR:AMEL(Xp22.2:Yp11.2): 2:1. SRY } \\
\text { (Yp11.31): positive. } \\
\text { DYS448(Yq11.223): negative. }\end{array}$ & $\begin{array}{l}\text { Hypospadia, congenital testicular } \\
\text { hypoplasia. His small penis was bent } \\
\text { towards the abdomen side, and } \\
\text { showed phimosis. }\end{array}$ & n.a. & n.a. \\
\hline 69433 & $\begin{array}{l}\text { female/ } \\
6 y\end{array}$ & PBL & $\begin{array}{l}45, X[13] / 46 \\
X,+\operatorname{mar}[8]\end{array}$ & $\min (Y)$ & MLPA: Y was abnormal. & $\begin{array}{l}\text { Pygmyism, asitia. H:106 cm, W:17.2 } \\
\text { kg, BW:2.9 kg. }\end{array}$ & n.a. & n.a. \\
\hline 61680 & male/29y & PBL & $\begin{array}{l}46, X, \operatorname{mar}[9] / \\
46, X Y[9]\end{array}$ & $\min (Y)$ with SRY & $\begin{array}{l}\text { AZF-d and -c regions: deleted. } \\
\text { STR:AMEL (Xp22.2:Yp11.2): 1:1. } \\
\text { SRY (Yp11.31): positive. }\end{array}$ & Azoospermatism & n.a. & n.a. \\
\hline 62091 & male/31y & PBL & $\begin{array}{l}45, X[8] / 46, X \\
\operatorname{mar}[7]\end{array}$ & $\min (Y)$ with SRY & $\begin{array}{l}\text { AZF-b, }-d \text { and }-c \text { regions: } \\
\text { deleted. STR:AMEL (Xp22.2: } \\
\text { Yp11.2): 1:1. SRY (Yp11.31): } \\
\text { positive. }\end{array}$ & Azoospermatism & n.a. & n.a. \\
\hline 77297 & male/26y & PBL & $46, X_{1}+$ mar & $\min (Y)$ with SRY & $\begin{array}{l}\text { AZF all regions: deleted. STR: } \\
\text { AMEL (Xp22.2:Yp11.2): 1:1. SRY } \\
\text { (Yp11.31): positive. }\end{array}$ & Azoospermatism & n.a. & n.a. \\
\hline 80794 & male/32y & PBL & $\begin{array}{l}46, X \\
+\operatorname{mar} 1[4] / 46, \\
X,+\operatorname{mar} 2[7] / \\
47, X,+\operatorname{mar} 3 \\
+ \text { mar4[12] }\end{array}$ & $\min (Y)$ with SRY & $\begin{array}{l}\text { AZF-b,-d and -c regions: deleted. } \\
\text { STR:AMEL (Xp22.2:Yp11.2): 1:1. } \\
\text { SRY (Yp11.31): positive. }\end{array}$ & Azoospermatism, infertile & n.a. & n.a. \\
\hline 98139 & male/28y & PBL & $46, X,+$ mar? & $\min (Y)$ with SRY & $\begin{array}{l}\text { AZF all regions: deleted. STR: } \\
\text { AMEL (Xp22.2:Yp11.2): 1:1. SRY } \\
\text { (Yp11.31): positive. }\end{array}$ & Infertile, azoospermatism. & n.a. & n.a. \\
\hline W01824 & male/31y & PBL & $\begin{array}{l}45, X[15] / 46 \\
X,+\operatorname{mar}[10]\end{array}$ & $\min (Y)$ without SRY & $\begin{array}{l}\text { AZF all regions: deleted. STR: } \\
\text { AMEL (Xp22.2:Yp11.2): 2:1. SRY } \\
\text { (Yp11.31): negative. }\end{array}$ & $\begin{array}{l}\text { Infertile, azoospermatism. He had } \\
\text { undergone remedial surgery for } \\
\text { hypospadias and cryptorchidism } \\
\text { when he was } 5 \text { years old. Magnetic } \\
\text { resonance imaging (MRI) showed } \\
\text { right spermatophore hypogenesis, } \\
\text { and left spermatophore containing } \\
\text { a mass. }\end{array}$ & n.a. & n.a. \\
\hline 150677 & $\begin{array}{l}\text { n.a./ } \\
\text { prenatal }\end{array}$ & $\mathrm{AF}$ & $\begin{array}{l}45, X[1] / 46, X \\
+\operatorname{mar}[19]\end{array}$ & $\min (Y)$ with SRY & $\begin{array}{l}\text { STR:AMEL (Xp22.2:Yp11.2): 1:1. } \\
\text { SRY (Yp11.31): positive. } \\
\text { DYS448(Yq11.223): positive. }\end{array}$ & $\begin{array}{l}\text { NIPT indicated abnormal } \\
\text { heterosomes. Gravida was G4P1, and } \\
\text { had nature labour twice. Spousal } \\
\text { AZF regions was normal. }\end{array}$ & $\begin{array}{l}38 / 46, X X \\
46, X Y\end{array}$ & de novo \\
\hline 162047 & $\begin{array}{l}\text { n.a./ } \\
\text { prenatal }\end{array}$ & $\mathrm{AF}$ & $\begin{array}{l}46, X \\
+\operatorname{mar}(Y ?)\end{array}$ & $\min (Y)$ with SRY & $\begin{array}{l}\text { STR:AMEL (Xp22.2:Yp11.2): 1:2. } \\
\text { SRY (Yp11.31): positive. } \\
\text { DYS448(Yq11.223): negative. }\end{array}$ & $\begin{array}{l}\text { NIPT indicated abnormal } \\
\text { heterosome. Gravida was G2P1. }\end{array}$ & 33/n.a. & n.a. \\
\hline $171276^{a}$ & $\begin{array}{l}\text { n.a./ } \\
\text { prenatal }\end{array}$ & $\mathrm{AF}$ & $\begin{array}{l}45, X[2] / 47, X \\
+ \text { mar1, } \\
+\operatorname{mar} 2[1] / 46, \\
X,+\operatorname{mar} 1[47]\end{array}$ & $\begin{array}{l}\operatorname{mar1:} \min (Y)(: \mathrm{p} 11.31 \\
\rightarrow \text { qter), mar2: inv } \\
\text { dul(Y)(q11.221 } \rightarrow \text { p11.31:: } \\
\text { p11.31 } \rightarrow \text { q11.221), first } \\
\text { report }\end{array}$ & $\begin{array}{l}\text { NGS: } \operatorname{dup}(Y)(p 11.31 \rightarrow q 11.221) \times 3 \text {, } \\
\text { del(Y)(q11.221 } \rightarrow q 12) \times 1, \text { mosaic } \\
\text { 45,X. STR: AMEL(X:Y): 1:2. SRY } \\
\text { (Yp11.31): positive. } \\
\text { DYS448(Yq11.223): negative. }\end{array}$ & $\begin{array}{l}\text { NT: } 4.7 \mathrm{~mm}(>3.0 \mathrm{~mm}) \text {. Gravida } \\
\text { underwent NGS in another hospital. }\end{array}$ & $24 / 46, X X$ & n.a. \\
\hline 69813 & male/6y & PBL & $47, X Y,+$ mar & $\begin{array}{l}\text { inv } \\
\operatorname{dup}(15)(q 11.2 \sim 13.3) \\
\operatorname{dul}(15) \mathrm{q}(13.3)\end{array}$ & $\begin{array}{l}\text { NGS: } \operatorname{dup}(15)(q 11.2 \rightarrow q 13.3) \times 4 \\
(8.2 \mathrm{Mb}) \text {, dup(15)q(13.3) } \times 3(1.6 \\
\text { Mb) }\end{array}$ & $\begin{array}{l}\text { Hypoevolutism, hypophrenia, } \\
\text { epilepsy. He could only say a few } \\
\text { words. His EEG demonstrated } \\
\text { epilepsy changes. }\end{array}$ & n.a. & n.a. \\
\hline W03987 & male/31y & PBL & $47, X Y,+$ mar & inv dup(15)(q11.2) & $\begin{array}{l}\text { NGS: polymorphism } \\
\text { dul(15)(q11.2)(22740001- } \\
\text { 23520000)×4 (0.78 Mb). AZF: } \\
\text { normal. SRY: positive. }\end{array}$ & Infertile, asthenospermia. & n.a. & n.a. \\
\hline W04210 & $\begin{array}{l}\text { female/ } \\
25 y\end{array}$ & PBL & $47, X X,+$ mar & $\min (15)(: q 11.2 \rightarrow q 13.1:)$ & $\begin{array}{l}\text { NGS: } \operatorname{dup}(15)(q 11.2 \rightarrow q 13.1) \times 3 \\
(5.64 \mathrm{Mb})\end{array}$ & $\begin{array}{l}\text { Hyperspasmia. She had } \\
\text { hyperspasmia for twenty years. Her } \\
\text { hyperspasmia occurred during } \\
\text { sleep, with tongue biting, foaming } \\
\text { at the mouth, and gatism, looking } \\
\text { like epilepsy. }\end{array}$ & n.a. & n.a. \\
\hline 70532 & male/2y & PBL & $47, X Y,+$ mar & inv dup(15)(q11.2) & MLPA: 3 points (two of SNRPN & Autism & n.a. & n.a. \\
\hline
\end{tabular}


Table 1 The information of 27 identified SSMC patients (Continued)

\begin{tabular}{|c|c|c|c|c|c|c|c|c|}
\hline $\begin{array}{l}\text { Patient } \\
\text { NO. }\end{array}$ & $\begin{array}{l}\text { Gender/ } \\
\text { age at } \\
\text { diagnosis }\end{array}$ & $\begin{array}{l}\text { Studied } \\
\text { material }\end{array}$ & Cytogenetics & Final result of the SSMC & Test methods and results & Clinical symptoms & $\begin{array}{l}\text { Age of } \\
\text { gravida/ } \\
\text { karyotypes } \\
\text { of parents }\end{array}$ & $\begin{array}{l}\text { De } \\
\text { novo/ } \\
\text { inherited }\end{array}$ \\
\hline & & & & & $\begin{array}{l}\text { and one of UBE3A) of } 15 q 11.2 \\
\text { were a heterozygous duplicated } \\
\text { mutation. }\end{array}$ & & & \\
\hline 83411 & $\begin{array}{l}\text { female/ } \\
5 y\end{array}$ & PBL & $47, \mathrm{XX},+$ mar & inv dup(15)(q11.2) & $\begin{array}{l}\text { MLPA: } 3 \text { points of } 15 q 11.2 \text { were } \\
\text { heterozygous duplicated } \\
\text { mutation. }\end{array}$ & $\begin{array}{l}\text { Hypoevolutism and mental } \\
\text { retardation. She could not sit on her } \\
\text { own at } 1 \text { year old and could not } \\
\text { walk at } 3 \text { years old. MRI showed } \\
\text { that her left lobus frontalis was } \\
\text { partly demyelinated. Ultrasound of } \\
\text { the heart revealed a ventricular } \\
\text { septal defect, left to right ventricle } \\
\text { shunt, wide coronary sinus, and } \\
\text { persistent left superior vena cava. }\end{array}$ & n.a. & n.a. \\
\hline 96862 & $\begin{array}{l}\text { female/ } \\
18 \mathrm{~m}\end{array}$ & PBL & $47, X X,+$ mar & inv dup(15)(q11.2) & $\begin{array}{l}\text { MLPA: } 3 \text { points of } 15 q 11.2 \text { were } \\
\text { heterozygous duplicated } \\
\text { mutation. }\end{array}$ & $\begin{array}{l}\text { Hypoevolutism. She could not walk } \\
\text { steadily or pick up things with her } \\
\text { fingers, and had poor } \\
\text { communication. MRI of the } \\
\text { cerebrum showed that both sides } \\
\text { of the hemisphere were not full. }\end{array}$ & n.a. & n.a. \\
\hline $92568^{\mathrm{a}}$ & $\begin{array}{l}\text { female/ } \\
12 \mathrm{y}\end{array}$ & PBL & $\begin{array}{l}45, X[7] / 46, X \\
+\operatorname{mar}[13]\end{array}$ & $\begin{array}{l}r(X)(:: p 11.23 \rightarrow q 21.1::) \\
\text { first report }\end{array}$ & $\begin{array}{l}\text { NGS: } 45, X[57 \%] / 46, X \\
r(X)(p 11.23 \rightarrow q 21.1)[43 \%]\end{array}$ & $\begin{array}{l}\text { She was suspected Turner } \\
\text { syndrome, and injected GH for } 1 \\
\text { year. }\end{array}$ & n.a. & n.a. \\
\hline W09834 & $\begin{array}{l}\text { female/ } \\
14 \mathrm{~m}\end{array}$ & PBL & $\begin{array}{l}45, X[4] / 46, X \\
+\operatorname{mar}[26]\end{array}$ & $\begin{array}{l}\min (X)(: p 11.2 \rightarrow q 13.2:) \\
\text { first report }\end{array}$ & $\begin{array}{l}\text { NGS: partly } 45, X: X(p t e r \rightarrow p 11.21) \\
\text { X1, X(q13.2 } \rightarrow \text { qter }) \times 1 . \text { SRY: } \\
\text { negative. }\end{array}$ & Turner syndrome. & n.a. & n.a. \\
\hline 61259 & $\begin{array}{l}\text { male/ } \\
57 d\end{array}$ & PBL & $47, \mathrm{XY},+$ mar & $\begin{array}{l}\text { inv } \\
\text { dup(18)(pter } \rightarrow \text { p11.21:: } \\
\text { p11.21 } \rightarrow \text { pter })\end{array}$ & $\begin{array}{l}\text { NGS: } \operatorname{dup}(18)(\mathrm{p} 11.32 \rightarrow \mathrm{p} 11.21) \times 4 \\
(15.3 \mathrm{Mb})\end{array}$ & $\begin{array}{l}\text { Neonatal feeding problem, } \\
\text { pneumonia. He had microcephaly, } \\
\text { low-set ears and often gazed look. }\end{array}$ & n.a. & n.a. \\
\hline 172168 & $\begin{array}{l}\text { female/ } \\
\text { prenatal }\end{array}$ & $\mathrm{AF}$ & $47, \mathrm{XX},+$ mar & $\begin{array}{l}\text { inv } \\
\text { dup(18)(pter } \rightarrow \text { p11.21:: } \\
\text { p11.21 } \rightarrow \text { pter })\end{array}$ & $\begin{array}{l}\text { NGS: } \operatorname{dup}(18)(\mathrm{p} 11.32 \rightarrow \mathrm{p} 11.21) \times 4 \\
\text { STR: normal. }\end{array}$ & $\begin{array}{l}\text { NIPT: the high risk of 18-trisomy } \\
\text { syndrome (Edwards syndrome). }\end{array}$ & $38 / 46, X X$ & n.a. \\
\hline $96932^{\mathrm{a}}$ & $\begin{array}{l}\text { female/ } \\
4 y\end{array}$ & PBL & $\begin{array}{l}45, X(21 \mathrm{ps}+) \\
{[14] / 46, X} \\
+ \text { mar, } \\
(21 \mathrm{ps}+)[6]\end{array}$ & $\begin{array}{l}\min (X), \min (Y), \text { first } \\
\text { report }\end{array}$ & $\begin{array}{l}\text { NGS: } 45, X[65 \%] / 46, X Y[17 \%] / 46, \\
X X[18 \%]\end{array}$ & $\begin{array}{l}\text { Hypoevolutism. She grew slowly } \\
\text { after birth, with W: } 12.5 \mathrm{~kg}, \mathrm{H}: 93 \mathrm{~cm} \text {, } \\
(\mathrm{H} / \mathrm{A} \leq 2 \mathrm{SD}) \text {. She had skin rash on } \\
\text { the face, webbed neck, and short } \\
\text { stature, looking like Turner } \\
\text { syndrome. Her bone age was } 3.5 \\
\text { years old, and } 4 \text { left carpals were } \\
\text { sclerotized. Ultrasound showed } \\
\text { vestige uterus and no ovary. }\end{array}$ & n.a. & n.a. \\
\hline $172990^{a}$ & $\begin{array}{l}\text { female/ } \\
\text { prenatal }\end{array}$ & $\mathrm{AF}$ & $47, X X,+$ mar & $\begin{array}{l}\min (9)(p t e r \rightarrow p 13.1:) \\
\text { first report }\end{array}$ & $\begin{array}{l}\text { NGS: dup(9)(p24.3 } \rightarrow \text { p13.1)×3. } \\
\text { STR: normal. }\end{array}$ & $\begin{array}{l}\text { NIPT indicated abnormal chrosome } \\
9 .\end{array}$ & $37 / 46, X X$ & n.a. \\
\hline $70963^{\mathrm{a}}$ & $\begin{array}{l}\text { female/ } \\
8 y\end{array}$ & PBL & $\begin{array}{l}47, X X \\
+\operatorname{mar}(1 \mathrm{qh}+) \\
{[18] / 46} \\
X X(1 \mathrm{qh}+)[12]\end{array}$ & $\begin{array}{l}\min (20)(: p 12.3 \rightarrow q 11.22:) \\
\text { first report }\end{array}$ & $\begin{array}{l}\text { NGS: mosaic duplication } \\
(20)(\text { p12.3 } \rightarrow \text { q11.22)×3 (20.1 Mb) }\end{array}$ & $\begin{array}{l}\text { Pygmyism,asitia. She had asitia and } \\
\text { was sickly; W: } 21.7 \mathrm{~kg}, \mathrm{H}: 115.5 \mathrm{~cm} \text {, } \\
\text { H/A } \leq-2 S D \text {. Her } 7 \text { left carpals were } \\
\text { sclerotized. Her mother's height was } \\
158 \mathrm{~cm} \text { and father's } 178 \mathrm{~cm} \text {. NGS } \\
\text { was done at another hospital. }\end{array}$ & n.a. & n.a. \\
\hline $160246^{a}$ & $\begin{array}{l}\text { female/ } \\
\text { prenatal }\end{array}$ & $\mathrm{AF}$ & $\begin{array}{l}\text { 160246: } 47 \\
X X,+\operatorname{mar}\end{array}$ & $\begin{array}{l}\min (11)(: q 23.3 \rightarrow \text { qter }) \\
\text { first report }\end{array}$ & $\begin{array}{l}\text { NGS: dup(11)(q23.3 } \rightarrow q 25) \times 3 \text {. STR: } \\
\text { normal. }\end{array}$ & $\begin{array}{l}\text { In 2016, her mother got pregnant } \\
\text { (numbered 160246). Ultrasound } \\
\text { showed that there was a fluid } \\
\text { sonolucent area in the nuchal } \\
\text { region of } 160246 . \text { NGS performed at } \\
\text { another hospital. In 2017, her } \\
\text { mother got pregnant again } \\
\text { (numbered 173026). The fetus } \\
\text { carried the same balanced } \\
\text { translocation, and his NGS results } \\
\text { were normal. }\end{array}$ & $\begin{array}{l}29 / 46, X X \\
t(11 ; \\
22)(q 23 ; \\
q 12) 46, X Y\end{array}$ & de novo \\
\hline 184290 & $\begin{array}{l}\text { male/ } \\
\text { prenatal }\end{array}$ & $A F$ & $47, \mathrm{XY},+$ mar & $\begin{array}{l}\operatorname{inv} \\
\operatorname{dup}(22)(q 11.1 \sim 11.21)\end{array}$ & $\begin{array}{l}\text { NGS:dup(22)(q11.21)×3(2.46 Mb), } \\
\text { dup(22)(q11.1 } \rightarrow \text { q11.21)×4. STR: } \\
\text { normal. }\end{array}$ & $\begin{array}{l}\text { NT: } 3.1 \mathrm{~mm} \text {. Gravida aborted a fetus } \\
\text { with congenital heart disease in } \\
2017 .\end{array}$ & $\begin{array}{l}32 / 46, X X \\
46, X Y\end{array}$ & de novo \\
\hline
\end{tabular}


Table 2 The information of 48 unidentified SSMC patients

\begin{tabular}{|c|c|c|c|c|c|c|c|}
\hline $\begin{array}{l}\text { Patient } \\
\text { NO. }\end{array}$ & $\begin{array}{l}\text { Gender/ } \\
\text { age at } \\
\text { diagnosis }\end{array}$ & $\begin{array}{l}\text { Studied } \\
\text { material }\end{array}$ & Cytogenetics & Test methods and results & Clinical symptoms & $\begin{array}{l}\text { Age of gravida/ } \\
\text { karyotypes of } \\
\text { parents }\end{array}$ & $\begin{array}{l}\text { De } \\
\text { novo/ } \\
\text { inherited }\end{array}$ \\
\hline 150234 & $\begin{array}{l}\text { male/ } \\
\text { prenatal }\end{array}$ & AF & $\begin{array}{l}47, X X,+\operatorname{mar}[23] / \\
46, X X[21]\end{array}$ & STR: normal & Diabetes of type II & 36/n.a. & n.a. \\
\hline 150693 & $\begin{array}{l}\text { female/ } \\
\text { prenatal }\end{array}$ & AF & $48, X X,+18,+$ mar & $\begin{array}{l}\text { STR: 18-trisomy syndrome (Ed- } \\
\text { wards syndrome) }\end{array}$ & $\begin{array}{l}\text { Down's syndrome screening: } \\
\text { high-risk. Advanced maternal } \\
\text { age. }\end{array}$ & 43/n.a. & n.a. \\
\hline 151434 & $\begin{array}{l}\text { male/ } \\
\text { prenatal }\end{array}$ & AF & $47, X Y,+$ mar & STR: normal. SRY: positive & $\begin{array}{l}\text { Ultrasound: ventricular septal } \\
\text { defect, small kidney. }\end{array}$ & $31 / 46, X X ; 46, X Y$ & de novo \\
\hline 153225 & $\begin{array}{l}\text { female/ } \\
\text { prenatal }\end{array}$ & AF & $\begin{array}{l}47, X X,+\operatorname{mar}[5] / 46, \\
X X[39]\end{array}$ & STR: normal & Ambryo develop delay & 28/n.a. & n.a. \\
\hline 161045 & $\begin{array}{l}\text { n.a./ } \\
\text { prenatal }\end{array}$ & AF & $\begin{array}{l}45, X[11] / 46, X \\
+\operatorname{mar}[21]\end{array}$ & STR: $45, X$ & $\begin{array}{l}\text { NIPT: abnormal heterosome, } \\
\text { NT:2.9 mm }\end{array}$ & 36/n.a. & n.a. \\
\hline 163110 & $\begin{array}{l}\text { male/ } \\
\text { prenatal }\end{array}$ & AF & $47, X Y,+$ mar & STR: normal. SRY: positive & $\begin{array}{l}\text { Cerebromedullary tube } \\
\text { anisotrophy. }\end{array}$ & $30 / 46, X X$ & n.a. \\
\hline 170574 & $\begin{array}{l}\text { n.a./ } \\
\text { prenatal }\end{array}$ & AF & $\begin{array}{l}45, X[30] / 46, X \\
+\operatorname{mar}[3]\end{array}$ & STR: $45, X$ & $\begin{array}{l}\text { Single umbilical artery (SUA), } \\
\text { seroperitoneum of fetus }\end{array}$ & 30/n.a. & n.a. \\
\hline 172376 & $\begin{array}{l}\text { n.a./ } \\
\text { prenatal }\end{array}$ & AF & $\begin{array}{l}46, X,+\operatorname{mar}[17] / 45 \\
X[12]\end{array}$ & STR: $45, X$ & $\mathrm{NT}>3 \mathrm{~mm}$ & $33 / 46, X X$ & n.a. \\
\hline 173060 & $\begin{array}{l}\text { female/ } \\
\text { prenatal }\end{array}$ & AF & $47, \mathrm{XX},+$ mar & STR: normal & $\begin{array}{l}\text { Down's syndrome screening: } \\
\text { high-risk }\end{array}$ & 26/n.a. & n.a. \\
\hline 180036 & $\begin{array}{l}\text { female/ } \\
\text { prenatal }\end{array}$ & AF & $\begin{array}{l}47, X X \\
+\operatorname{mar}[1](S C) / 46 \\
X X[35]\end{array}$ & STR: normal & Oligohydramnios. & 30/n.a. & n.a. \\
\hline 180748 & $\begin{array}{l}\text { female/ } \\
\text { prenatal }\end{array}$ & AF & $\begin{array}{l}47, X X \\
+\operatorname{mar}[3](M C) / 46 \\
X X[22]\end{array}$ & STR: normal & Twins & 28/n.a. & n.a. \\
\hline 181010 & $\begin{array}{l}\text { female/ } \\
\text { prenatal }\end{array}$ & AF & $\begin{array}{l}47, X X,+\operatorname{mar}[1] / 46, \\
X X[29]\end{array}$ & NIPT: low risk. STR: normal & Ventricular septal defect & 37/n.a. & n.a. \\
\hline 183584 & $\begin{array}{l}\text { male/ } \\
\text { prenatal }\end{array}$ & AF & $\begin{array}{l}47, X Y,+\operatorname{mar}[1] / 46, \\
X Y[29]\end{array}$ & STR: normal & $\begin{array}{l}\text { Down's syndrome screening: } \\
\text { high-risk(1/346). }\end{array}$ & 31/n.a. & n.a. \\
\hline 184082 & $\begin{array}{l}\text { female/ } \\
\text { prenatal }\end{array}$ & AF & $\begin{array}{l}47, X X,+\operatorname{mar}[1] / 46, \\
X X[29]\end{array}$ & STR: normal & $\begin{array}{l}\text { Down's syndrome screening: } \\
\text { high-risk }\end{array}$ & 26/n.a. & n.a. \\
\hline 184172 & $\begin{array}{l}\text { male/ } \\
\text { prenatal }\end{array}$ & AF & $\begin{array}{l}47, X Y \\
+\operatorname{mar}[1](\mathrm{SC}) / 46, \\
\mathrm{XY}[24]\end{array}$ & $\begin{array}{l}\text { NGS: dup(11)(p15.3 } \rightarrow \text { p15.3) } \times 3 \\
\text { dup(6)(p12.32)(32400000- } \\
\text { 32780000)×3 }\end{array}$ & NT: $2.5 \mathrm{~mm}$ & 27/n.a. & n.a. \\
\hline A1045 & $\begin{array}{l}\text { female/ } \\
\text { prenatal }\end{array}$ & UCB & $47, \mathrm{XX},+$ mar & & $\begin{array}{l}\text { Develop delay for one } \\
\text { month. }\end{array}$ & 30/n.a. & n.a. \\
\hline 61200 & male/32y & PBL & $\begin{array}{l}47, X Y,+\operatorname{mar}[6] / 36 \\
X Y[13]\end{array}$ & AZF: normal. SRY: positive & Infertile. & n.a. & n.a. \\
\hline 61397 & male/24y & PBL & $47, X Y,+$ mar & AZF: normal. SRY: positive & $\begin{array}{l}\text { Azoospermatism, } \\
\text { hyperprolactinemia. }\end{array}$ & n.a. & n.a. \\
\hline 62254 & female/3y & PBL & $47, \mathrm{XX},+$ mar & MLPA: normal & Global developdelay & n.a. & n.a. \\
\hline 63001 & male/29y & PBL & $47, \mathrm{XY},+$ mar & AZF: normal. SRY: positive & Asthenospermia & n.a. & n.a. \\
\hline 63411 & female/22y & PBL & $47, X X,+$ mar & & $\begin{array}{l}\text { The mother of a patient with } \\
\text { develop delay. }\end{array}$ & n.a. & n.a. \\
\hline 65676 & female/3y & PBL & $\begin{array}{l}48, X X,+21, \\
+\operatorname{mar}[13] / 47, X X,+ \\
21[7]\end{array}$ & & Heart malformation & n.a. & n.a. \\
\hline 67979 & female/9y & PBL & $\begin{array}{l}46, X,+\operatorname{mar}[14] / 45, \\
X[11]\end{array}$ & & Runtishness & n.a. & n.a. \\
\hline 69235 & $\begin{array}{l}\text { female/12 } \\
\mathrm{m}\end{array}$ & PBL & $\begin{array}{l}46, X,+\operatorname{mar}[8] / 45, \\
X[12]\end{array}$ & MLPA: X was abnormal & Developdelay & n.a. & n.a. \\
\hline 72699 & male/3y & PBL & $\begin{array}{l}48, X Y,+ \text { mar1 } \\
+ \text { mar2 }\end{array}$ & MLPA: normal & Autism. & n.a. & n.a. \\
\hline
\end{tabular}


Table 2 The information of 48 unidentified SSMC patients (Continued)

\begin{tabular}{|c|c|c|c|c|c|c|c|}
\hline $\begin{array}{l}\text { Patient } \\
\text { NO. }\end{array}$ & $\begin{array}{l}\text { Gender/ } \\
\text { age at } \\
\text { diagnosis }\end{array}$ & $\begin{array}{l}\text { Studied } \\
\text { material }\end{array}$ & Cytogenetics & Test methods and results & Clinical symptoms & $\begin{array}{l}\text { Age of gravida/ } \\
\text { karyotypes of } \\
\text { parents }\end{array}$ & $\begin{array}{l}\text { De } \\
\text { novo/ } \\
\text { inherited }\end{array}$ \\
\hline 73431 & male/10y & PBL & $\begin{array}{l}48, X Y,+ \text { mar1 } \\
+ \text { mar2 }\end{array}$ & SRY: positive & Astigmia and hypometropia. & n.a. & n.a. \\
\hline 73940 & female/59y & PBL & $47, X X,+$ mar & & n.a. & n.a. & n.a. \\
\hline 7300 & male/33y & PBL & $47, X Y,+$ mar & $\begin{array}{l}\text { NGS: No obvious abnormal was } \\
\text { detected. AZF: normal. SRY: } \\
\text { positive. }\end{array}$ & $\begin{array}{l}\text { Asthenospermia, } \\
\text { teratospermia. }\end{array}$ & n.a. & n.a. \\
\hline 80039 & male/33y & PBL & $47, X Y,+$ mar & $\begin{array}{l}\text { NGS: A } 0.46 \mathrm{Mb} \text { section deleted } \\
\text { in } 6 \mathrm{q} 12 \text {, no pathopoiesia } \\
\text { information. }\end{array}$ & Infertile,asthenospermia. & n.a. & n.a. \\
\hline 81882 & female/15y & PBL & $\begin{array}{l}46, X, \operatorname{mar}[11] / 45 \\
X[9]\end{array}$ & & Primary amenorrhea & n.a. & n.a. \\
\hline 85773 & male/32y & PBL & $47, X Y,+$ mar & $\begin{array}{l}\text { NGS: No obvious abnormal was } \\
\text { detected. }\end{array}$ & Infertile, azoospermatism. & n.a. & n.a. \\
\hline 90074 & female/9 m & PBL & $\begin{array}{l}45, X[8] / 46, X \\
+\operatorname{mar}[19]\end{array}$ & NGS: $45, X$ & Hypoevolutism & n.a. & n.a. \\
\hline 91473 & female/20y & PBL & $\begin{array}{l}46, X,+\operatorname{mar}[11] / 45, \\
X[10]\end{array}$ & SRY: negtive & $\begin{array}{l}\text { Primary amenorrhea.Vestigial } \\
\text { uterus. }\end{array}$ & n.a. & n.a. \\
\hline 92243 & female/34y & PBL & $\begin{array}{l}47, X X,+\operatorname{mar}[19] / \\
46, X X[13]\end{array}$ & $\begin{array}{l}\text { NGS: No obvious abnormal was } \\
\text { detected. }\end{array}$ & Infertile & n.a. & n.a. \\
\hline 92638 & female/25y & PBL & $\begin{array}{l}45, X,+\operatorname{mar}[1] / 46 \\
X X[16] / 47, X X \\
+\operatorname{mar}[3]\end{array}$ & & Infertile & n.a. & n.a. \\
\hline 93162 & n.a./9 m & PBL & $\begin{array}{l}45, X[11] / 46, X \\
+\operatorname{mar}[9]\end{array}$ & & Gynandromorphism & n.a. & n.a. \\
\hline 96704 & female/2y & PBL & $\begin{array}{l}45, X[17] / 46, X \\
+\operatorname{mar}[13]\end{array}$ & & Pygmyism & n.a. & n.a. \\
\hline 97858 & male/30y & PBL & $47, X Y,+m a r$ & AZF: normal. SRY: positive & Infertile, azoospermatism. & n.a. & n.a. \\
\hline W00311 & female/30y & PBL & $47, X X,+m a r$ & $\begin{array}{l}\text { NGS: A } 0.14 \mathrm{Mb} \text { section deleted } \\
\text { in 2q32.1, no pathopoiesia } \\
\text { information. }\end{array}$ & $\begin{array}{l}\text { G1P0 embryonic stop } \\
\text { develop at } 11 \text { weeks. }\end{array}$ & n.a. $/ 47, X X_{1}+$ mar & maternal \\
\hline W00880 & female/53y & PBL & $47, X X,+m a r$ & & W00311's mother & n.a. & n.a. \\
\hline W02523 & female/21y & PBL & $\begin{array}{l}47, X X,+\operatorname{mar}[2] / 46 \\
X X[23]\end{array}$ & & $\begin{array}{l}\text { G4P0. Habitual abortion, } \\
\text { arrested embryo. }\end{array}$ & n.a. & n.a. \\
\hline W03572 & female/7 m & PBL & $\begin{array}{l}47, X X,+\operatorname{mar}[1] / 46, \\
X X[29]\end{array}$ & & Develop delay. & n.a. & n.a. \\
\hline W06115 & female/30y & PBL & $47, X X,+m a r$ & & G3P1. Arrested embryo twice. & n.a. & n.a. \\
\hline W06490 & female/29y & PBL & $\begin{array}{l}47, X X,+\operatorname{mar}[26] / \\
46, X X[12]\end{array}$ & & G2P0. Arrested embryo twice. & n.a. & n.a. \\
\hline W07384 & male/30y & PBL & $\begin{array}{l}47, X Y,+\operatorname{mar}[3] / 46, \\
X Y[36]\end{array}$ & & $\begin{array}{l}\text { Spouse had one time } \\
\text { hydatidiform mole. }\end{array}$ & n.a. & n.a. \\
\hline W13749 & female/4y & PBL & $47, X X,+m a r$ & & Developmental retardation. & n.a. & n.a. \\
\hline W13804 & male/18 m & PBL & $\begin{array}{l}45, X[12] / 46, X \\
+\operatorname{mar}[18]\end{array}$ & & Hypospadia & n.a. & n.a. \\
\hline W14357 & female/28y & PBL & $47, X X,+$ mar & & Pregnant preparation & n.a. & n.a. \\
\hline
\end{tabular}

Abbreviations: PBL peripheral blood, AF amniotic fluid, UCB umbilical cord blood, y year, $m$ month, $d$ day, n.a not available, NIPT non-invasive prenatal testing, NT nuchal translucency

sSMCs from chromosome 15

The sSMCs of six patients were derived from chromosome 15. NGS identified duplications on chromosome 15 for patients 69813 and W03987 (Fig. 3A, B). MLPA revealed that patients 70532, 83411, and 96862 had a heterozygous duplicated mutation at 15q11.2 (Fig. 3DF). These five patients carried sSMCs derived from inv $\operatorname{dul}(15)$. The sSMC of patient W04210 was from min(15) (Fig. 3C). Five of these cases showed clinical features of Dup15q syndrome (e.g., hypoevolutism or autism). In 


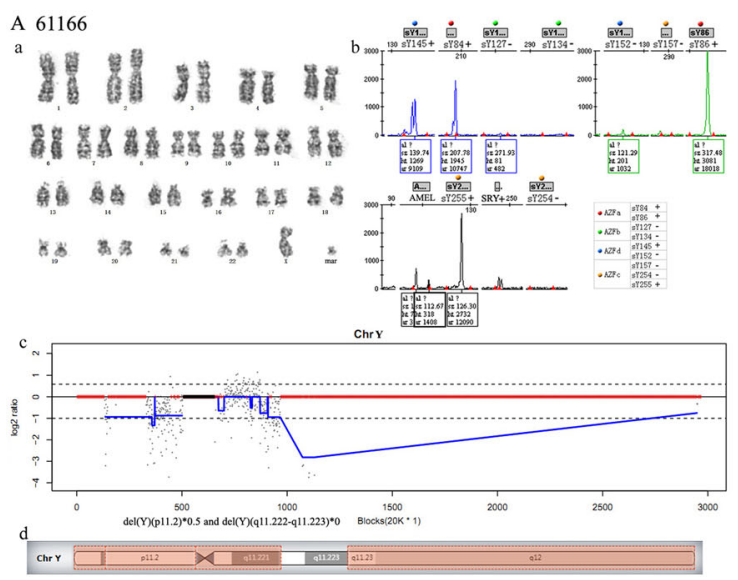

II If If of if |f If

H $\|$ If or \&

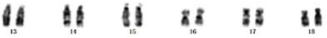

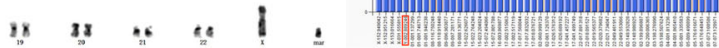

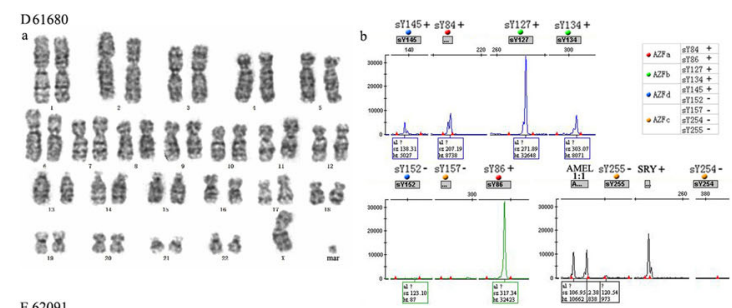

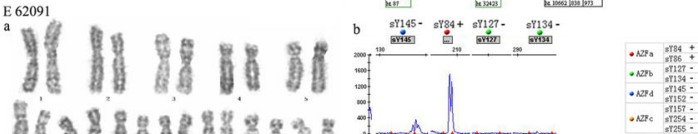

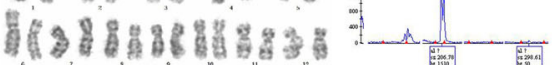

bิ

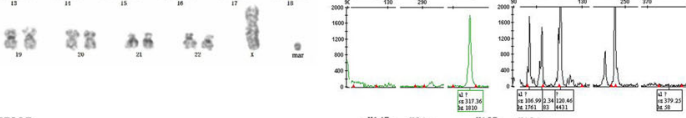

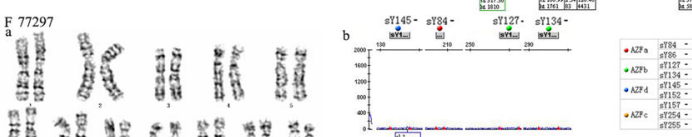

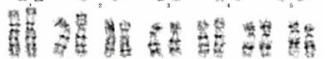

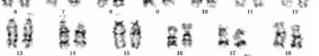

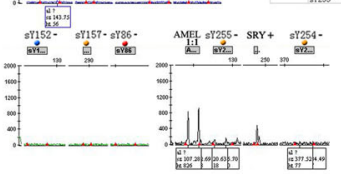

$\mathrm{G} 80794$

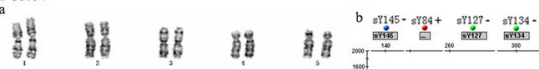

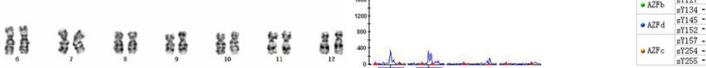

.

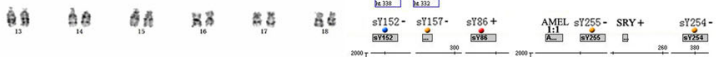

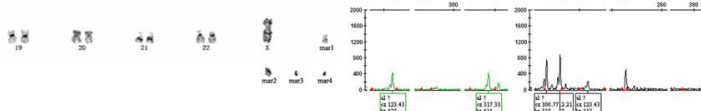

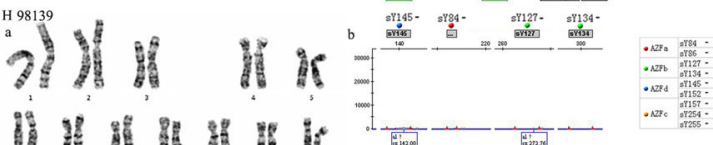

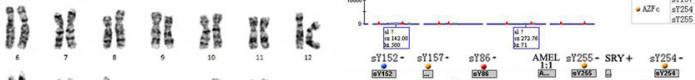

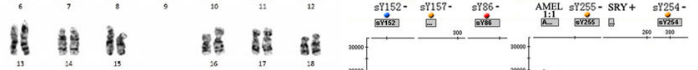

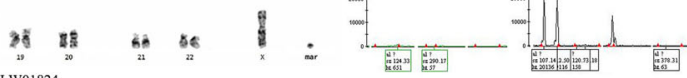

al He

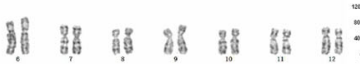

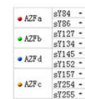

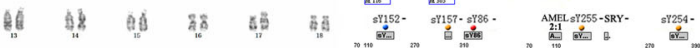

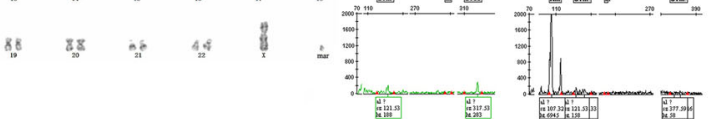

If

${ }_{\mathrm{a}}^{\mathrm{K}} 162047$

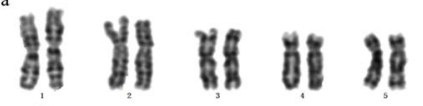

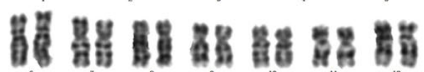

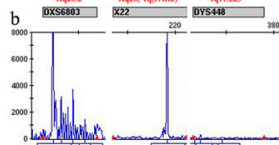

南

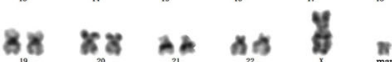

L 171276

\&)

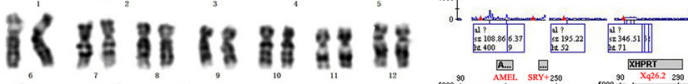

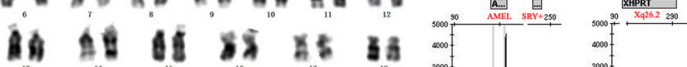

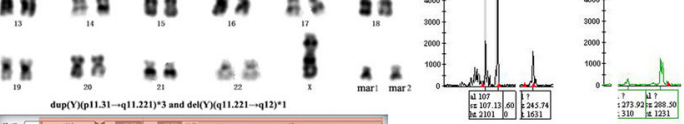

Fig. 2 (See legend on next page.) 
(See figure on previous page.)

Fig. 2 Cytogenetic and molecular results for patients carrying sSMCs derived from min(Y). (A) 61166: (a) The karyotype was detected by G-banding. (b) AZF-b, AZF-d, and AZF-c regions were deleted. The SRY was positive. (c) NGS identified two deletions on chromosome Y. (d) The location of the sSMC on chromosome $Y$ is highlighted in red. (B) W02938: (a) The karyotype was mosaic. (b) STR AMEL(X:Y) was 2:1, DYS448(Yq11.223) was not detected (negative), but SRY was positive. (C) 69433: (a) The karyotype was mosaic. (b) MLPA detected an abnormal Y in "Y-002.889246". The data for patients 61680, 62091, 77297, 80794, 98139 and W01824 were presented separately from (D) to (I). (a) The karyotypes were revealed by G-banding. (b) STR analysis detected deletions in the AZF regions. The date of 150677, 162047 and 171276 were presented separately from (J) to (L). (a) The karyotypes were revealed by G-banding. (b) STR detected X and Y chromosomes. (L) 171276: (c) The location of the SSMC on chromosome Y is highlighted in red

contrast, case W03987 with inv dup(15)(q11.2) was polymorphic without the features of Dup15q syndrome.

\section{sSMCs from chromosome $X$}

The sSMCs of two patients were derived from chromosome X. These patients showed characteristics of Turner syndrome. NGS indicated that the sSMC of patient 92568, which was mosaic $(45, \mathrm{X} / 46, \mathrm{X},+\mathrm{mar})$, might be from $r(\mathrm{X})$ (Fig. 4A). The sSMC of patient W09834 was partial 45, $\mathrm{X}$ and composed of $\min (\mathrm{X})$ (Fig. 4B).

\section{sSMCs from chromosome 18}

The sSMCs of patient 61259 and fetus 172168 were derived from inv dul(18) (Fig. 5A, B). NGS showed that they had the genotype $\operatorname{dup}(18)(\mathrm{p} 11.32 \rightarrow \mathrm{p} 11.21) \times 4$. It has been reported that the clinical symptoms are likely isochromosome 18p [i(18p)] syndromes or tetrasomy $18 \mathrm{p}$ syndrome, which feature neonatal feeding problems, hypoevolutism, and high risk of infections $[5,6]$.

\section{sSMCs from other chromosomes}

NGS showed that patient 96932 had a complex sSMC that might be derived from $\min (\mathrm{X})$ and $\min (\mathrm{Y})$ (Fig. 6A). This patient displayed similar characteristics to Turner syndrome. The sSMC of fetus 172990 was derived from $\min (9)$ (Fig. 6B). The sSMC of patient 70963, who showed compound features of partial trisomy 20p and 20q11.22 duplication syndrome with pygmyism and asitia, was derived from $\min (20)$ (Fig. 6C). The sSMC of fetus 160246 was derived from $\min (11)$ (Fig. 7A-a, b). When her mother got pregnant again, the fetus carried the same balanced translocation (Fig. 7A-c). The sSMC of fetus 184290 was derived from inv. dup(22) (Fig. 7B).

\section{sSMCs of unknown chromosomal origin}

Although several techniques were used to identify the origin of the different sSMCs, 48 patients could not be diagnosed (Table 2). Amniotic fluid samples containing sSMCs were submitted for STR analysis, and only seven sSMCs were identified. From karyotyping, these unidentified sSMCs were classified into three groups (Fig. 8). Group I sSMCs consisted of inverted duplicated chromosomes. Those in group II were likely minute chromosomes, while those in group III looked like ring chromosomes.

\section{Discussion}

In this study, we identified the origins of 27 sSMCs, of which, eight sSMCs are being reported for the first time (Table 1). Of the 27 defined sSMC origins, 12 were derived from the $\mathrm{Y}$ chromosome and two from the $\mathrm{X}$ chromosome. The infertile patients showed azoospermia, and their original Y sSMCs were detected. Azoospermia factor (AZF), which is located on the long arm of $\mathrm{Y}$ (Yq11.23), regulates spermatogenesis [7]. These patients had deletions of AZF-a region (the Sertoli cell-only syndrome), AZF-b region (sperm-maturation-arrest syndrome), or all AZF regions resulting in azoospermia.

Table 3 The results of AZF

\begin{tabular}{|c|c|c|c|c|c|c|c|c|}
\hline Regions & & 61166 & 61680 & 62091 & 77297 & 80794 & 98139 & W01824 \\
\hline \multirow[t]{2}{*}{ AZFa } & sY84 & + & + & + & - & + & - & - \\
\hline & sY86 & + & + & + & - & + & - & - \\
\hline \multirow[t]{2}{*}{ AZFb } & sY127 & - & + & - & - & - & - & - \\
\hline & sY134 & - & + & - & - & - & - & - \\
\hline \multirow[t]{2}{*}{ AZFd } & sY145 & + & + & - & - & - & - & - \\
\hline & sY152 & - & - & - & - & - & - & - \\
\hline \multirow[t]{3}{*}{ AZFC } & sY157 & - & - & - & - & - & - & - \\
\hline & sY254 & - & - & - & - & - & - & - \\
\hline & sY255 & + & - & - & - & - & - & - \\
\hline SRY & & + & + & + & + & + & + & - \\
\hline
\end{tabular}




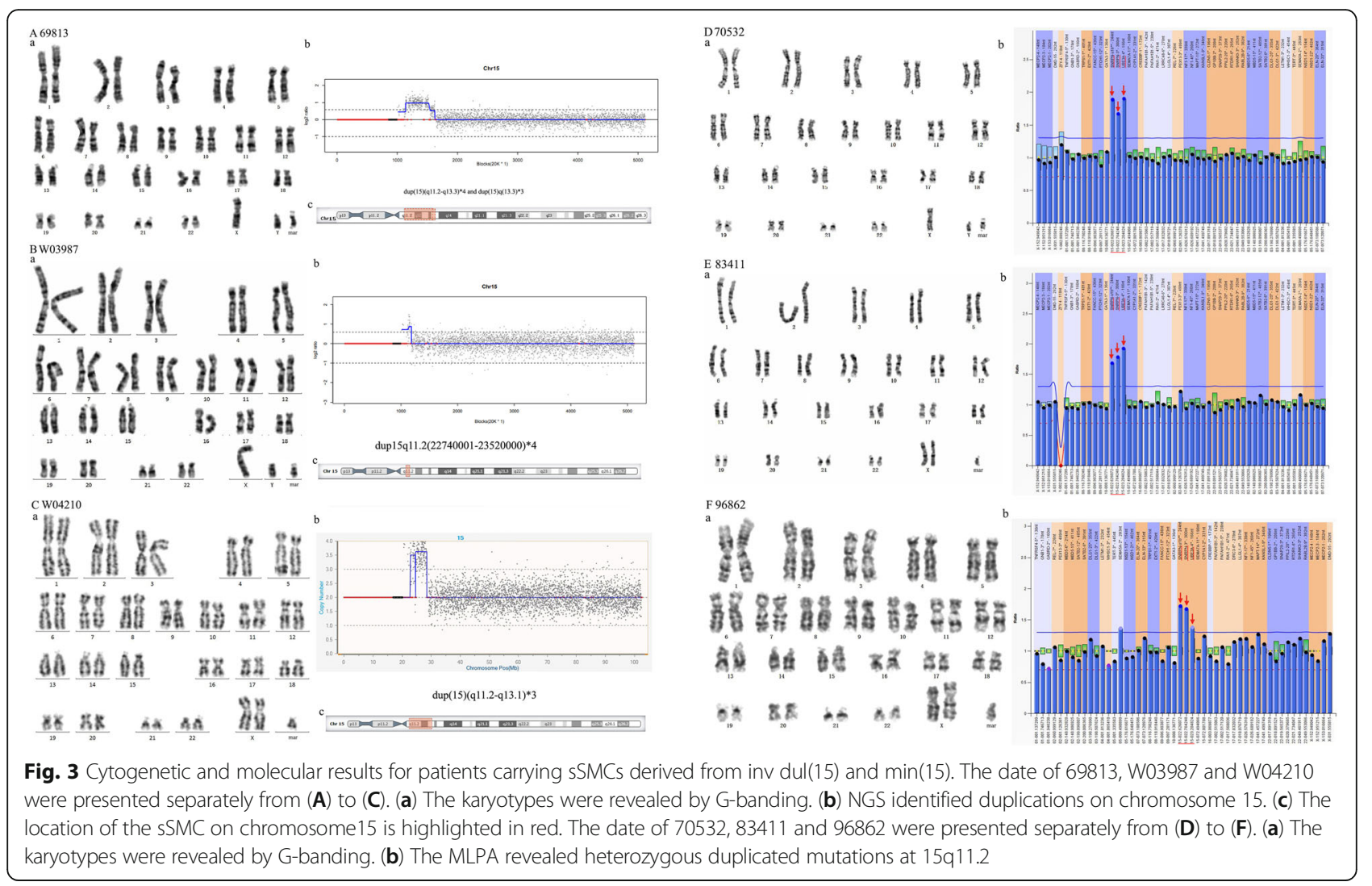

Thus, artificial insemination with donor sperm or adoption was suggested for clinical management. The pediatric patients carrying sSMCs from $\min (\mathrm{Y})$ or chromosome $\mathrm{X}$ or complex sSMCs from $\min (\mathrm{X})$ and $\min (\mathrm{Y})$ had similar characteristics to Turner syndrome; however, they had different phenotypes depending on their sSMC origins. The short arm of $\mathrm{X}$ harbors the short stature-homeobox gene (SHOX on Xp22.33) and lymphogenic gene (forkhead box P3, FOXP3 on

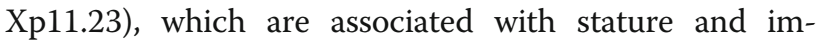
munodeficiency or polyendocrinopathy [8]. Patient W09834 with $\min (\mathrm{X})$ had a loss of FOXP3 and an immunological problem. A similar sSMC derived from $\mathrm{r}(\mathrm{X})(: \mathrm{p} 11.21 \rightarrow \mathrm{q} 13.1::)$ was reported in craniofrontonasal syndrome (CFNS) [9]. The methyl-CpG binding protein2 gene (MECP2 on Xq28) is located on the long arm of $X$. This gene correlates with RETT syndrome and the premature ovarian failure gene POF (POF1: Xq21 $\rightarrow$ qter, POF2: $\mathrm{Xq} 13.3 \rightarrow \mathrm{Xq} 21.1)[10]$. As the $\min (\mathrm{X})$ from patient W09834 (:p11.2 $\rightarrow$ q13.2:) and $r(X)$ from patient 92568 (::p11.23 $\rightarrow \mathrm{q} 21.1::)$ did not contain SHOX and $M E C P 2$, both patients had growth retardation and a high risk of RETT syndrome. As they had the part of POFs, so being attention to ovarian function. Patient 96932 had a complex sSMC from $\min (\mathrm{X})$ and $\min (\mathrm{Y})$, resulting in a high risk of type II germ cell tumors [11, 12]. All the pediatric patients were recommended for individualized treatment according to their genotyperelated phenotypes.

Our sSMC patients with the 47,XN,+mar karyotype typically had special duplication syndrome, and six sSMCs were identified from inv dul(15). The region $15(\mathrm{q} 11.2 \rightarrow \mathrm{q} 13.3)$ is a known hot breakpoint. This region harbors the GABAAR genes, the paternal gene $S N R P N$, and the maternal gene $U B E 3 A$, which regulate central neural system development and function [13]. It was rare that two neocentric sSMCs derived from inv dup(18) had the same duplication fragment. There may be a hot breakpoint located at 18(p11.21). In region 18p, approximately 67 genes can contribute to the phenotypes, including AFG3L2, MC2R, and TGIF1, which are associated with developmental disorders $[5,6]$. So, when taking care of patient 61259, pay attention to artificial feeding, avoiding infections, and evaluating affected organs and systems. The region of 20 (p12.3 $\rightarrow \mathrm{q} 11.22)$ comprises more than 2 hundred genes. Duplication of $J A G 1$, $B T B D 3$, and FLRT3, or ASXL1 induces Alagille syndrome, neurological dysfunction or chromatin remodeling [14, 15]. Patient 70963 with the genotype $\min (20)(: \mathrm{p} 12.3 \rightarrow \mathrm{q} 11.22:)$ showed moderate symptoms due to $60 \%$ mosaic.

The identification of sSMCs is vital in prenatal diagnosis. Of the 75 sSMC cases from this study, 23 were from fetuses with intrauterine growth retardation or abnormal 


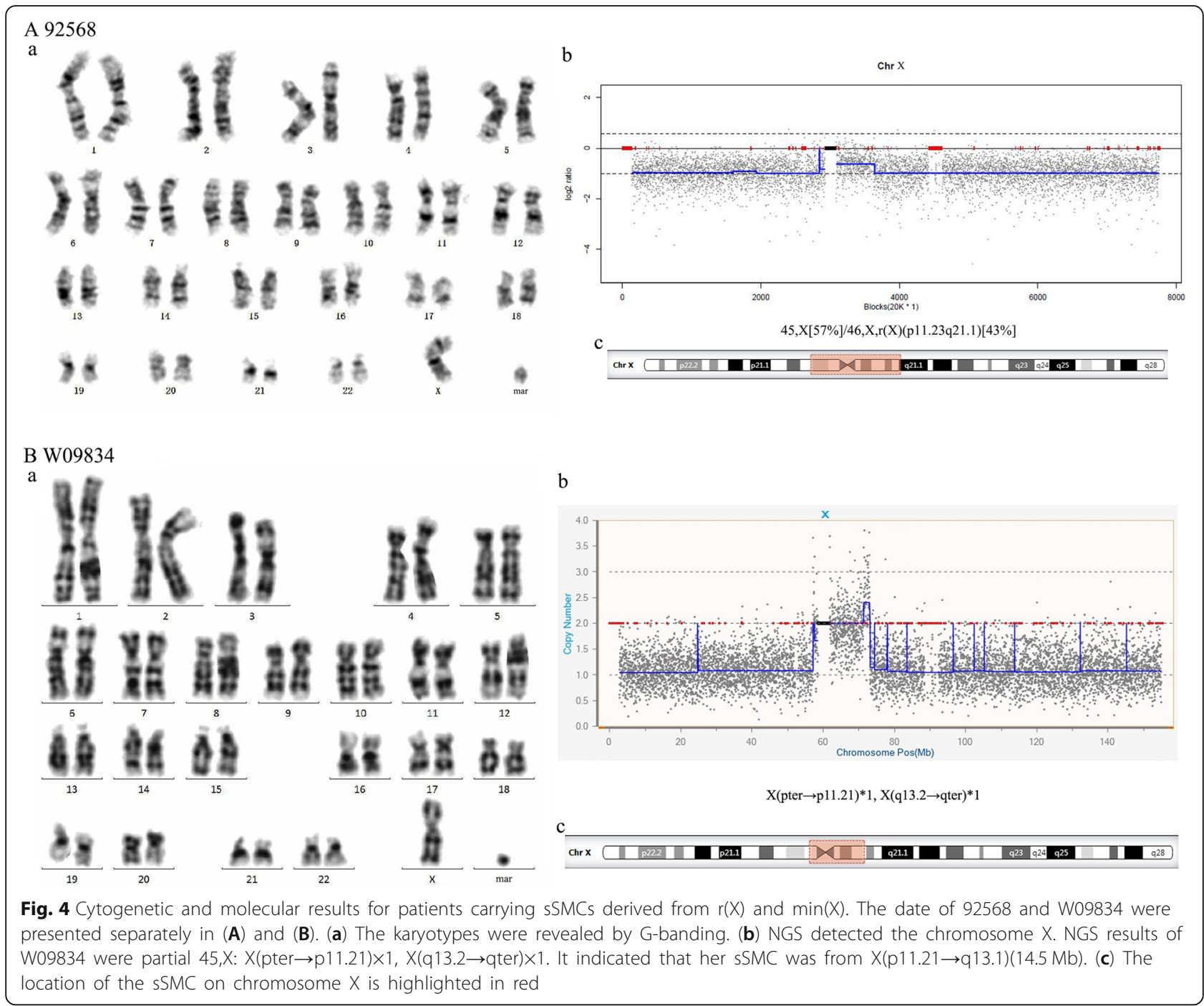

ultrasonic structure, and seven fetal sSMC cases were found to have $\mathrm{Y}, 18,9,11$, or 22 chromosomal origins. However, most sSMCs failed to define the original chromosome. Three fetal sSMCs from the Y chromosome needed careful evaluation. If the sSMCs correlated with androgyneity or AZF deletion, it was better to complete the pregnancy. However, if a fetus had an inv dup(18) genotype, termination of the pregnancy was suggested because of the $\mathrm{i}(18 \mathrm{p})$ syndromes. Fetus 172990 had a duplicated region $9(\mathrm{p} 24.3 \rightarrow \mathrm{p} 13.1)$ that correlated with $9 \mathrm{p}$ duplication syndrome, which contains a potential autism spectrum disorder (ASD) and a normal IQ individual region [16, 17]. The sSMC of fetus 160246 was de novo and arose from a maternal balanced translocation $\mathrm{t}(11 ; 22)(\mathrm{q} 23 ; \mathrm{q} 12)$, leading to three copies of $11(\mathrm{q} 23.3 \rightarrow \mathrm{q} 25)$. The $\mathrm{sSMC}$ derived from the inv $\operatorname{dup}(22)$ chromosome was also de novo. The fetus carrying this sSMC had similar regions to the 22q11.2 duplication syndrome (22DupS), which usually produces birth defects, such as congenital heart disease, hearing loss, hypophrenia, or high risk of psychosis (including autism) $[18,19]$. A similar sSMC arising from inv $\operatorname{dup}(22)(\mathrm{q} 11.1 \sim 11.2)$ was reported with mild clinical signs [20].

Most sSMCs in fetuses are de novo, but a few are inherited from their parents. Thus, prenatal diagnosis and genetic counseling are critical. In our department, parents are asked to fill out a form to collect genetic information. Amniotic fluid is then submitted for both karyotyping and STR analysis. If an sSMC is diagnosed, further testing (e.g., NGS) is suggested, and the karyotypes of the parents are requested. If the parents are sSMC or translocation carriers, the fetus should take further testing. Preimplantation genetic screening (PGS) 


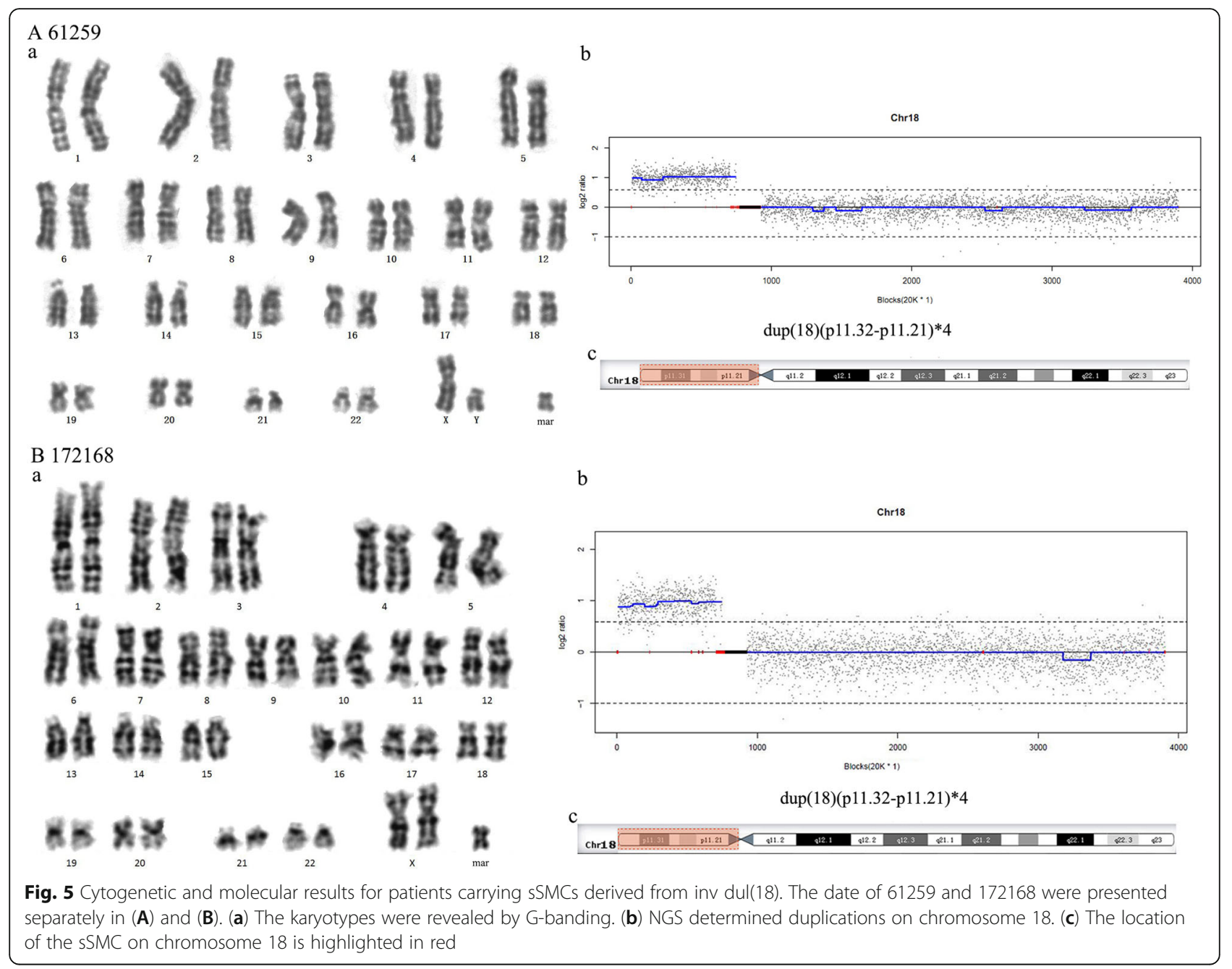

and preimplantation genetic diagnosis (PGD) would help reduce the chances of miscarriage.

Although several sequencing-related techniques were used in our study, there were still $30 \mathrm{sSMCs}$ for which pathogenic information could not be generated. It is possible that the sequencing primers did not cover the SSMC regions in the MLPA or STR (AZF) methods. Also, inverted duplicated chromosomes (acrocentric chromosomes), isochromosomes, or minute chromosomes (centromere-nearby regions) might not have been detected by NGS due to the highly repeated sequences at the centromere regions, which will be improved in read depth, inducing read pair, split pair, or assembly-based analysis of NGS. Thus, a set of efficient techniques should be developed for further sSMC identification.

\section{Conclusions}

In summary, the sSMCs of the study patients were different in origin, size, replication times, affected genes, and mosaicism levels. Thus, their clinical manifestations varied. This study detailed the comprehensive characterization of 27 sSMCs. Eight of these sSMCs are being reported here for the first time, which provides additional information for sSMC research. The identification of sSMCs could reveal genotype-phenotype correlations and integrate genomic data into clinical care.

\section{Methods}

\section{Patients' collection}

This research investigated 74,266 patients' specimens in our department from 2015 to 2018, including 50,794 peripheral bloods from adults, 6,350 peripheral bloods from pediatrics, 14,759 amniotic fluids, and 2,363 cord bloods. 75 sSMC carriers were diagnosed by karyotyping (Tables 1 and 2), containing 52 live births, and 23 fetuses. Some of them took further detection (e.g., NGS, MLPA, or STR). Then we identified the molecular component of $27 \mathrm{sSMC}$ cases. They were compared with the information in http://cs-tl.de/DB/CA/sSMC/0-Start. html. These retrospective studies were approved by the 


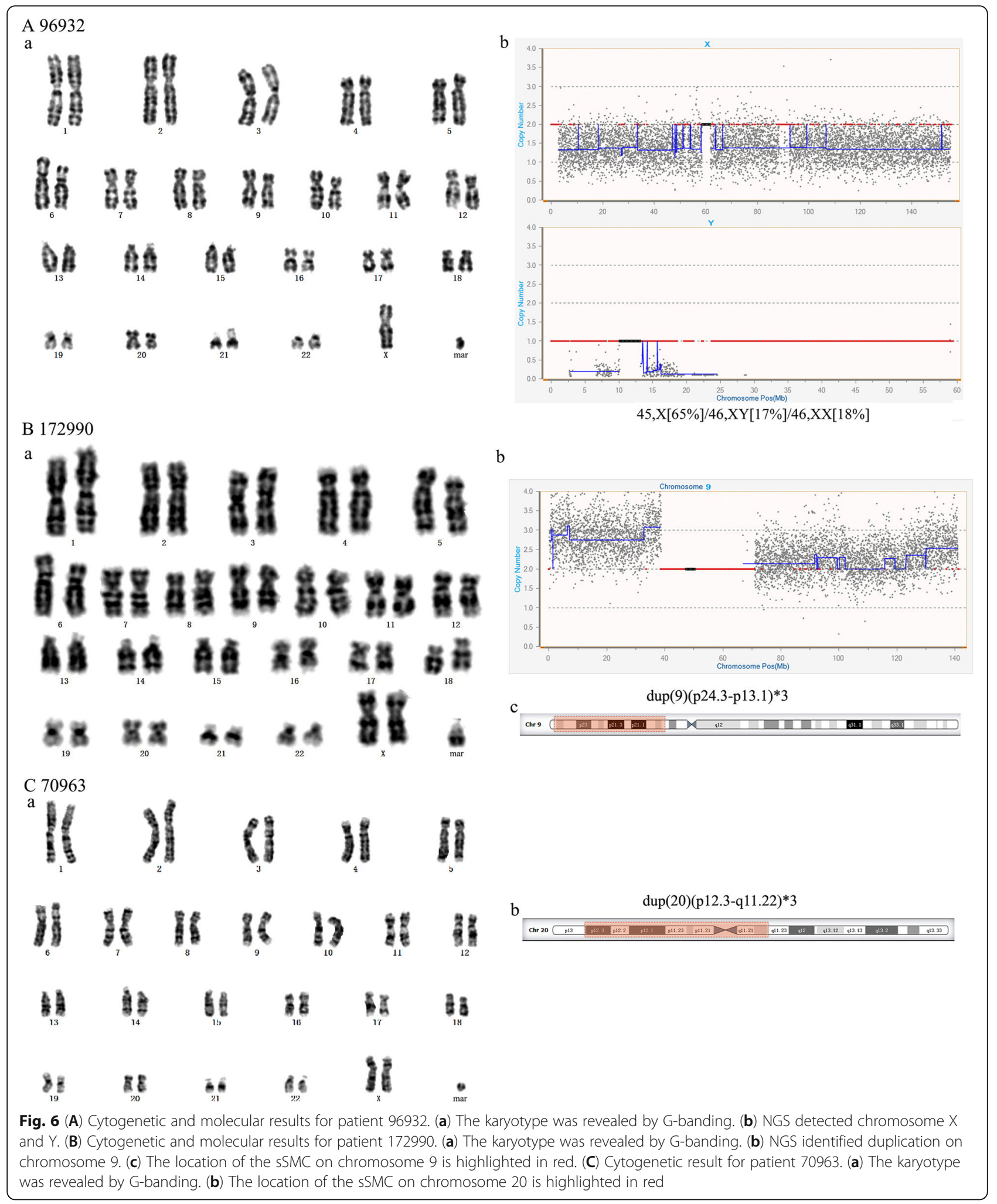




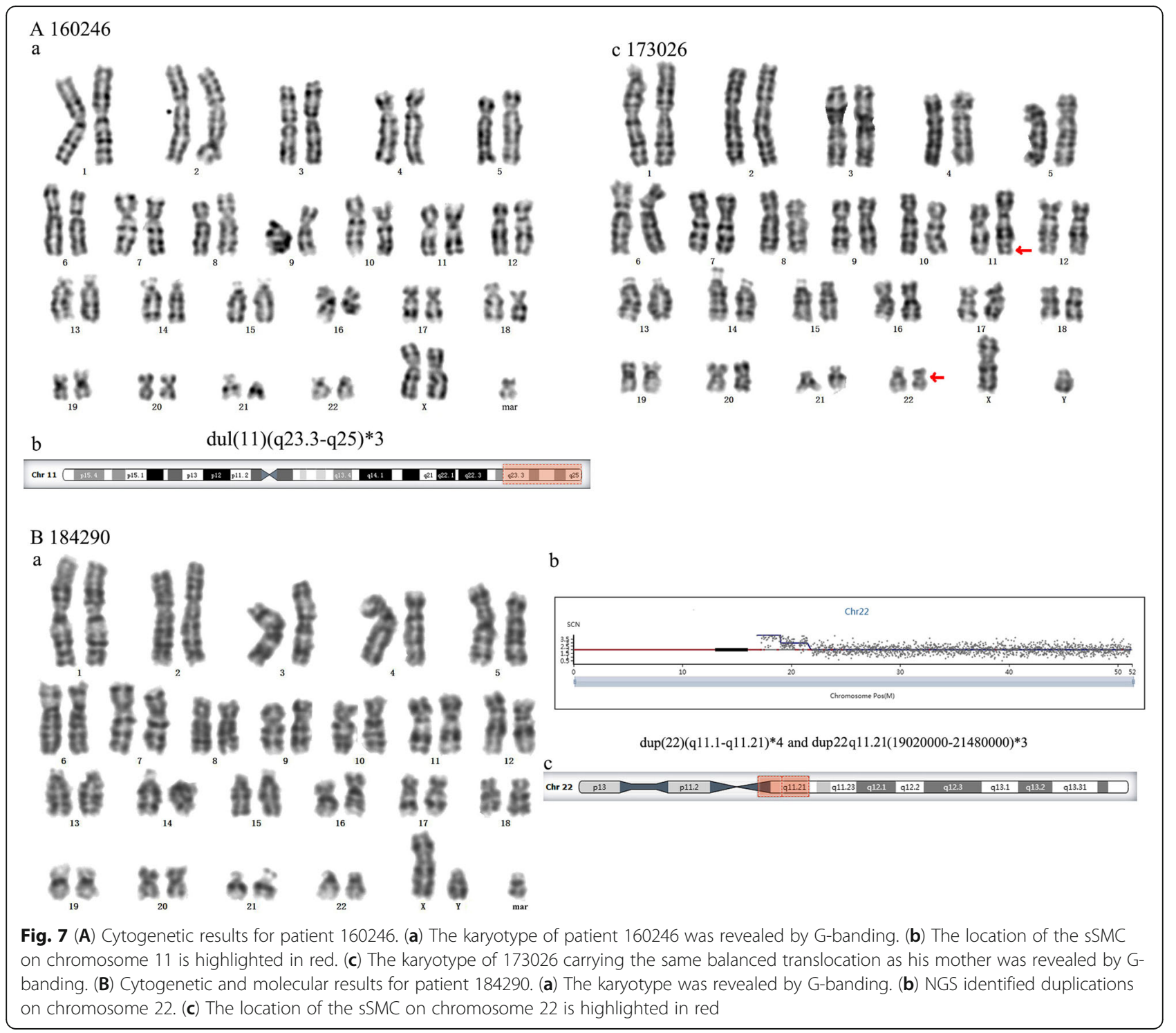

ethical commission of the Shengjing Hospital of China Medical University (NO.2019PS423K).

\section{Chromosome karyotyping}

Patients' peripheral blood and amniotic fluid samples were cultured, harvested, and stained with Giemsa (Gbanded) (at the resolution of approximately 300-400 bands) following the standard protocols. Then scanned in Lieca Cyto Vision (German) and analyzed according to the ISCN 2013.

\section{STR and AZF detection}

In our department first-generation sequencing (FGS) (3730 DNA Analyzer, Singapore) was used to detect STR on five chromosomes $(13,18,21, \mathrm{X}$, and $\mathrm{Y}$ in amniotic fluid), AZF(Yq11.2) and SRY(Yp11.31) of Y (in azoospermia adult). DNA was extracted by kit (BioBase, Chengdu, China) in Auto-Pure32A (ALL SHENG, Hangzhou, China), mixed with sequencing primers and Tag DNA Polymerase (Transgen, Beijing, China), and then did PCR (S1000 Thermal Cycler) and sequenced.

\section{MLPA}

MLPA was performed in FGS (3730 DNA Analyzer, Singapore) by the protocol of "SALSA ${ }^{\circ} \mathrm{MLPA}^{\circ} \mathrm{P} 245$ Microdeletion Syndromes-1" kit (MRC Holland, Amsterdam, the Netherlands). The preparation of DNA samples was same as STR. MLPA could suggest 23 kinds of deletion or duplication syndrome. Sequencing primers were illustrated in protocol, including one of Xp21.1, three of Xq28, three of 15q11.2 (one UBE3A probe and two SNRPN probes), and one (Y-fragment S0135- 


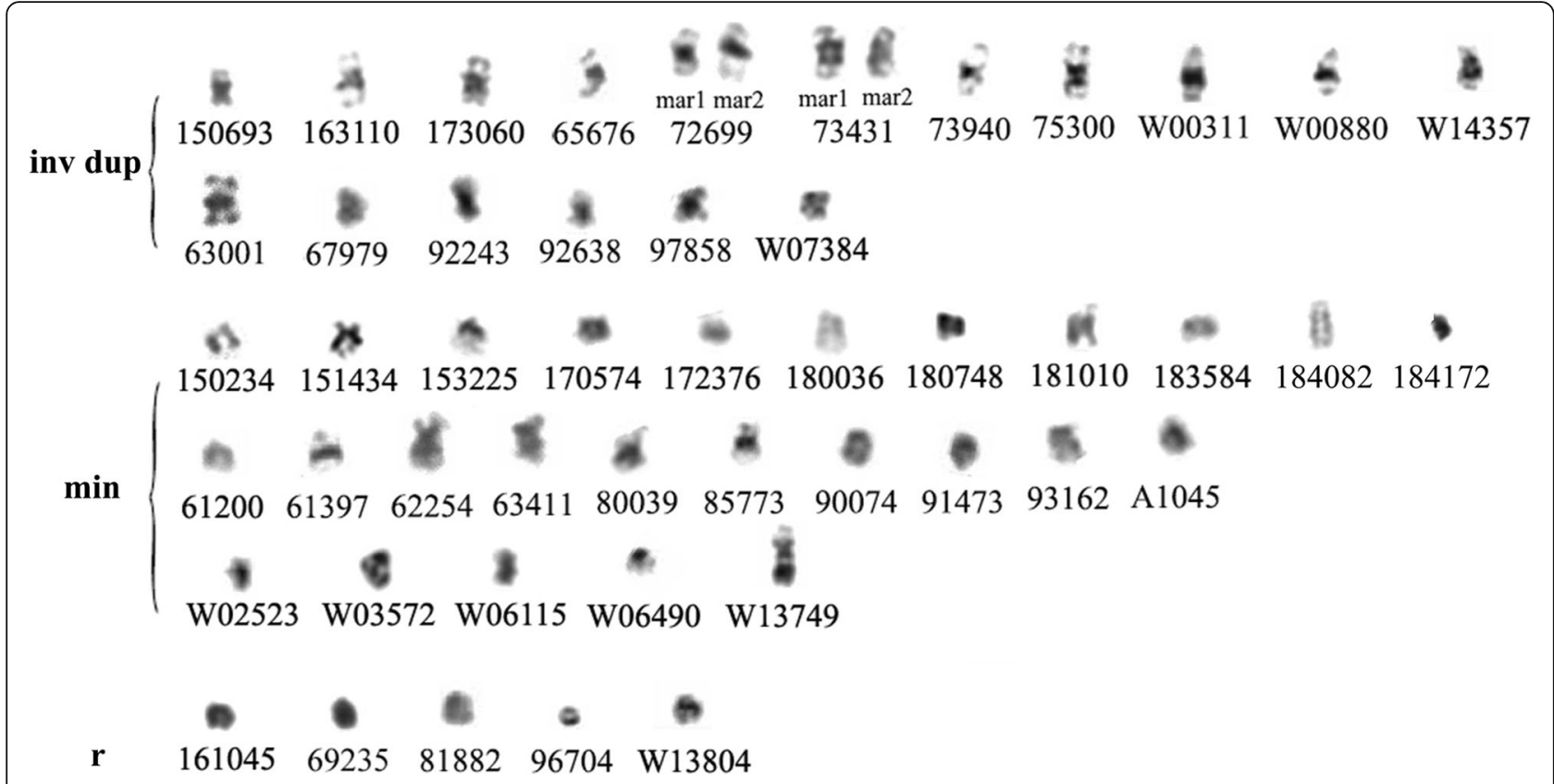

Fig. 8 From karyotyping, unidentified SSMCs were classified into three groups. I: inverted duplicated chromosomes; II: minute chromosomes; III: ring chromosomes

L16766) for the Y chromosome. MLPA data were presented with ratio.

\section{Next generation-sequencing}

NGS was performed in accordance with the protocols of a commercial NGS sequencing kit (Berry Genomics, Hangzhou, China). DNA samples were prepared by the extract kit (Axygen, MA, USA), purified and enriched library, then sequenced in the Illumina NextSeq CN500 (Berry Genomics, Hangzhou, China). Sequencing data were analyzed with Software VI (Berry Genomics, Hangzhou, China) in h19 database, blasted and searched information of disease in DGV, DECIPHER, OMIM, UCSC and Pubmed. Data were presented with $\log 2$ ratio or copy numbers (SCN).

\section{Abbreviations \\ SSMC: Small supernumerary marker chromosomes; NGS: Next-generation sequencing; MLPA: Multiplex ligation-dependent probe amplification; \\ STR: Short tandem repeats; AZF: Azoospermia factor; FGS: First-generation sequencing; NT: Nuchal translucency; NIPT: Non-invasive prenatal testing; MGS: Mixed gonadal dysgenesis; ART: Assisted reproductive technology; PGS: Preimplantation genetic screening; PGD: Preimplantation genetic diagnosis; LCRs: Low copy repeats}

\section{Acknowledgments}

We would like to thank the staffs of Department of Clinical Genetics for supporting this study.

\section{Authors' contributions}

YanZ and TL conceived and designed the study. HS participated in data analysis and performed statistics. TL, GC, YZ, MQ, XL and WC performed the genetic diagnosis. TL analyzed data and drafted the manuscript. YanZ and TL and revised it. All authors read and approved the final manuscript.

\section{Funding}

This work was supported by the following funding:

1. National Key R\&D Program of China (project no. 2016YFC1000700 and 2016YFC1000702) to Yanyan Zhao.

2. Free Researcher Foundation of Shengjing Hospital (project no. 201503) to Yanyan Zhao.

3. National Natural Science Foundation of China (project no. 81001091) to Tingting Li.

4. Doctor Starting Foundation of Liaoning province (project no. 2015010827301) to Haiquan Sang.

\section{Availability of data and materials}

The datasets used or analyzed during the current study are available from the corresponding author on reasonable request.

\section{Ethics approval and consent to participate}

This retrospective study was approved by the ethical commission of the Shengjing Hospital of China Medical University (NO.2019PS423K).

\section{Consent for publication}

Not applicable.

\section{Competing interests}

The authors have no conflicts of interest to declare.

\section{Author details}

${ }^{1}$ Department of Clinical Genetics, Shengjing Hospital of China Medical University, 36 Sanhao Street, Heping District, Shenyang 110003, China. ${ }^{2}$ Department of General Surgery, the Fourth Affiliated Hospital of China Medical University, Shenyang, China.

Received: 1 April 2020 Accepted: 10 June 2020

Published online: 14 July 2020

\section{References}

1. Jafari-Ghahfarokhi H, Moradi-Chaleshtori M, Liehr T, et al. Small supernumerary marker chromosomes and their correlation with specific syndromes. Adv Biomed Res. 2015;4:140. 
2. Hochstenbach R, Nowakowska B, Volleth M, et al. Multiple small supernumerary marker chromosomes resulting from maternal meiosis I or II errors. Mol Syndromol. 2016;6(5):210-21.

3. Bartels I, Schlueter G, Liehr T, et al. Supernumerary small marker chromosome (SMC) and uniparental disomy 22 in a child with confined placental mosaicism of trisomy 22: trisomy rescue due to marker chromosome formation. Cytogenet Genome Res. 2003;101:103-5.

4. Kerkhof J, Schenkel LC, Reilly J, et al. Clinical validation of copy number variant detection from targeted next-generation sequencing panels. J Mol Diagn. 2017;19(6):905-20.

5. Sebold C, Roeder E, Zimmerman M, et al. Tetrasomy 18p: report of the molecular and clinical findings of 43 individuals. Am J Med Genet A. 2010; 152A(9):2164-72.

6. Bawazeer S, Alshalan M, Alkhaldi A, et al. Tetrasomy 18p: case report and review of literature. Appl Clin Genet. 2018:11:9-14.

7. Yu XW, Wei ZT, Jiang YT, et al. Y chromosome azoospermia factor region microdeletions and transmission characteristics in azoospermic and severe oligozoospermic patients. Int J Clin Exp Med. 2015; 15;8(9):14634-14646.

8. Yu TY, Lin HS, Chen PL, et al. An isodicentric X chromosome with gonadal dysgenesis in a lady without prominent somatic features of Turner's syndrome. A case report. J Formos Med Assoc. 2015;114(1):77-80.

9. Evers $C$, Jungwirth MS, Morgenthaler J, et al. Craniofrontonasal syndrome in a male due to chromosomal mosaicism involving EFNB1: further insights into a genetic paradox. Clin Genet. 2014;85(4):347-53.

10. Chauhan P, Jaiswal SK, Lakhotia AR, et al. Molecular cytogenetic characterization of two turner syndrome patients with mosaic ring $X$ chromosome. J Assist Reprod Genet. 2016;33(9):1161-8.

11. Bertelloni $\mathrm{S}$, Dati $\mathrm{E}$, Valetto $\mathrm{A}$, et al. Long-term growth hormone treatment in a boy with $45, X / 46, X, i d i c(Y p)$ mixed gonadal dysgenesis: comparison with growth pattern of an untreated patient. Hormones (Athens). 2015;14(1):1427 .

12. Fukui $\mathrm{S}$, Watanabe $\mathrm{M}$, Yoshino $\mathrm{K}$, et al. $45, \mathrm{X}$ mosaicism with $\mathrm{Y}$ chromosome presenting female phenotype. J Pediatr Surg. 2015;50(7):1220-3.

13. Bonuccelli A, Valetto A, Orsini A, et al. Maternally derived 15q11.2-q13.1 duplication in a child with Lennox-Gastaut-type epilepsy and dysmorphic features: clinical-genetic characterization of the family and review of the literature. Am J Med Genet A. 2017;173(2):556-60.

14. Bartolini L, Sartori S, Lenzini E, et al. De novo trisomy 20p characterized by array comparative genomic hybridization: report of a novel case and review of the literature. Gene. 2013;524(2):368-72.

15. Avila M, Kirchhoff M, Marle N, et al. Delineation of a new chromosome 20q11.2 duplication syndrome including the ASXL1 gene. Am J Med Genet A. 2013;161A(7):1594-8.

16. Abu-Amero KK, Hellani AM, Salih MA, et al. A de novo marker chromosome derived from $9 p$ in a patient with $9 p$ partial duplication syndrome and autism features: genotype-phenotype correlation. BMC Med Genet. 2010;11:135.

17. Hulick PJ, Noonan KM, Kulkarni S, et al. Cytogenetic and array-CGH characterization of a complex de novo rearrangement involving duplication and deletion of $9 \mathrm{p}$ and clinical findings in a 4-month-old female. Cytogenet Genome Res. 2009;126(3):305-12.

18. Wenger TL, Miller JS, DePolo LM, et al. 22q11.2 duplication syndrome: elevated rate of autism spectrum disorder and need for medical screening. Mol Autism. 2016;7:27.

19. Nguyen LT, Fleishman R, Flynn E, et al. 22q11.2 microduplication syndrome with associated esophageal atresia/tracheo-esophageal fistula and vascular ring. Clin Case Rep. 2017;5(3):351-6.

20. Lohmann L, Chelloug N, Rosales B, et al. Dicentric marker derived from chromosome 22 associated with mild clinical signs: a case report. Prenat Diagn. 2000;20(2):156-8.

\section{Publisher's Note}

Springer Nature remains neutral with regard to jurisdictional claims in published maps and institutional affiliations.

Ready to submit your research? Choose BMC and benefit from:

- fast, convenient online submission

- thorough peer review by experienced researchers in your field

- rapid publication on acceptance

- support for research data, including large and complex data types

- gold Open Access which fosters wider collaboration and increased citations

- maximum visibility for your research: over $100 \mathrm{M}$ website views per year

At BMC, research is always in progress.

Learn more biomedcentral.com/submissions 